\title{
Testing of Aspheric Surfaces with Computer Generated Holograms
}

\author{
H.Tiziani, B.Packroß, G.Schmidt \\ Institut für Technische Optik, \\ Universität Stuttgart, Pfaffenwaldring 9, 7000 Stuttgart 80, FRG
}

\section{Summary}

Aspherical surfaces are becoming more important and can even be mass producted. There is a need for flexible test methods of high accuracy. The paper describes the combination of a computer generated holograms with a partially compensating lens as a powerful tool. An example for testing a steep aspheric surface will be given.

\section{Introduction}

Interferometric techniques in combination with digital fringe analysis are frequently used for testing optical components and surfaces. Problems occur when testing aspherical surfaces with classical two-beam interferometry for the following reasons. The fringe density becomes very high and the resolution of conventional detectors e.g. CCD-arrays is too small to resolve the fringe pattern. Possible techniques are $2 \lambda$-interferometry and shearing interferometry, mechanical and optical tasters, and null-lens arrangements. Some specific disadvantages are however

- reduced sensitivity $(2 \lambda$, shearing)

- damage of the surface (mechanical taster)

- no flexibility, expensive, difficult to test (null-lens).

The use of computer generated holograms (CGHs) in combination with a partially compensating lens is an alternative.

CGHs can be used for absolute testing of a wide range of aspheric surfaces. There are a number of publications concerning this subject [1-15]. For symmetrical optical systems to be tested, in-line (Gabor-type) and off-axis CGHs can be used [11,14]. Although in-line holograms have some advantages like simpler adjustment and the possibility of compensating stronger asphericities, off-axis holograms are mostly used. The spatial separation of their diffraction orders allows a simple filtering and avoids the overlap of different orders, which leads to difficulties to test the central portion of the aspherical surface when using in-line holograms. 
The principle of a measurement will be described in the following. The interferometric set-up will be described as well as the calculation and manufacturing of the off-axis holograms and the digital fringe analysis for the evaluation of the interference pattern.

\section{Test procedure with the Twyman-Green interferometer}

For testing aspherical surfaces with computer generated holograms a two-beam interferometer in which both beams pass through the CGH is used. In this way inhomogenities of the holographic storing device are compensated. Our two-beam interferometer of the Twyman-Green type is shown in figure 1. The plane wave leaving the beam expander is separated by the beam-splitter into reference and test wave. The plane reference mirror is piezo-driven, which is necessary for the phase stepping fringe analysis to be described later.

A precision lens to be used for testing spherical surfaces is placed in the test arm of the interferometer. It was specially designed to test surfaces with short radius and high numerical apcrture. An additional optical system between beam-splitter and precision lens may be required. Such a system allows to image the test surface onto the hologram to adapt the aperture and to compensate some of the asphericity of the test wavcfront. The CGH is mounted in the interferometer exit. The diffraction orders of the off-axis hologram are separated spatially in order to block unwanted orders by a iris diaphragm in the focal plane of a collimating lens. Figure 2 shows a section of the Twyman-Green interferometer. For a perfect aspheric surface the hologram transforms the aspheric test wave into a plane wave in the first diffraction order (figure 3). Shape errors lead to wave deformations. The undiffracted plane reference wave remains plane when passing through the hologram in the zero order. Both waves are superimposed by tilting the reference mirror. For the fringe analysis the hologram plane is imaged onto the CCD-chip of a TV-camera connected to a frame grabber in a personal computer.

\section{Calculation and manufacturing of the $\mathrm{CGH}$}

The calculation of the CGH starts with the data of the surface to be tested : radius, shape factor or conic constant, higher aspheric coefficients and diameter. For the ray-tracing this surface is considered as a reflecting last surface in the test arm of the Twyman-Green interferometer. The resulting wavefront in the hologram plane is obtained by ray tracing of an incident plane wave through the known collimating lens. After reflection at the surface under test the wavefront is computed back through the lens and the cubic beamsplitter to the hologram plane. For a first calculation the best fit sphere of the aspheric is evaluated. Subtracting its radius from the back focal length of the lens leads to an approximately best position of the test surface. This position is then varied by optimization routines to obtain a wavefront with minimum slope and 
aberration in the hologram plane. Three conditions have to be fulfilled for testing the whole diameter :

- the slope of the wavefront does not exceed a maximum given by the resolution of the photoplotter

- all rays reflected at the surface under test reach the hologram plane at a height less than the maximum plotable CGH diameter

- monotonously increasing height of rays in the hologram plane

If one of these conditions is not satisfied, either an additional compensating system has to be designed and inserted, or the testable diameter is limited up to that one, where the above conditions are satisfied.

The ray tracing delivers the optical path difference of the particular rays leading to the wavefront. For practical reasons (computation time) up to 50 rays are calculated, much less than the number of pixels to be plotted in a CGH. Thus the wavefront has to be developed into a polynomial. The number of terms depends on the slope of the wavefront. For rotational symmetric surfaces only even terms are necessary leading to even powers of the height in the hologram plane for instance. Their coefficients link the ray tracing and the plot program.

The wavefront in the hologram plane can be written as :

$$
W_{h}(r)=A_{2} r^{2}+A_{4} r^{4}+A_{6} r^{6}+A_{8} r^{8}+A_{10} r^{10}
$$

where $\mathrm{r}^{2}=\mathrm{x}^{2}+\mathrm{y}^{2}$ with $\mathrm{x}, \mathrm{y}$ are coordinates in the hologram plane and the A's are coefficients of the polynomial series.

For an off-axis hologram a carrier frequency is introduced by tilting the wavefront by $\alpha$, which yields to a resulting transmission distribution for the CGH :

$$
\mathrm{T}(\mathrm{x}, \mathrm{y})=0.5\left\{1+\cos \left[\frac{2 \pi}{\lambda}\left(\mathrm{W}_{\mathrm{h}}(\mathrm{x}, \mathrm{y})+\mathrm{x} \cdot \sin \alpha\right)\right]\right\}
$$

In the real measuring procedure errors of the test apparatus need to be compensated. Manufacturing inaccuracies and misalignments of the collimating lens, the compensating lens and other optical components lead to wavefront errors $\mathrm{W}_{\mathrm{c}}(\mathrm{x}, \mathrm{y})$. For this purpose the interferometer is calibrated with a very good sphere, expressing the wavefront in Zernike polynomials and adding them into the hologram calculation :

$$
\mathrm{T}(\mathrm{x}, \mathrm{y})=0.5\left\{1+\cos \left[\frac{2 \pi}{\lambda}\left(\mathrm{W}_{\mathrm{h}}(\mathrm{x}, \mathrm{y})+\mathrm{x} \cdot \sin \alpha+\mathrm{W}_{\mathrm{c}}(\mathrm{x}, \mathrm{y})\right)\right]\right\}
$$

This function will be calculated column by column and plotted on-line. 
For generating a $\mathrm{CGH}$ we use an Optronics $\mathrm{P}-1700$ drum plotter with a maximum plot size of $250 \times 250 \mathrm{~mm}^{2}$ and a minimum spot size of $12,5 \mu \mathrm{m}$. This resolution is not sufficient for a carrier frequency of 50 lines $/ \mathrm{mm}$, which is necessary for compensating high wavefront slopes of steep aspherics. For the final use in the interferometer a photographic reduction of approximately a factor 6,4 on Agfa Millimask plates or on photoresist is done.

\section{Digital fringe analysis}

The fringe pattern contains information about the shape error of the surface under test. Thus an evaluation of the fringe pattern leads to a description of the surface crror. Many different algorithms can be used for fringe analysis. Especially suited are phase-stepping techniques with at least three interferograms obtained by shifting the phase of the reference mirror [16-18].

We normally use two different algorithms to calculate the wavefront. The first one uses four interferograms and allows a very fast calculation of the wavefront phase. The four measured intensity distributions can be written as :

$$
\begin{aligned}
& I_{1}(x . y)=I_{0}(x, y)\left[1+m \cdot \cos \left(\varphi(x, y)-\frac{3}{2} \Delta\right)\right] \\
& I_{2}(x . y)=I_{0}(x, y)\left[1+m \cdot \cos \left(\varphi(x, y)-\frac{1}{2} \Delta\right)\right] \\
& I_{3}(x . y)=I_{0}(x, y)\left[1+m \cdot \cos \left(\varphi(x, y)+\frac{1}{2} \Delta\right)\right] \\
& I_{4}(x . y)=I_{0}(x, y)\left[1+m \cdot \cos \left(\varphi(x, y)+\frac{3}{2} \Delta\right)\right]
\end{aligned}
$$

where $\mathrm{I}_{0}$ mean intensity, $\mathrm{m}$ fringe contrast, $\varphi$ phase of wavefront, $\Delta$ introduced phase shift. Using a phase shift of $\Delta=\frac{\pi}{2}$ we obtain the phase $\varphi$ as :

$$
\varphi=\arctan \left[\frac{I_{4}-I_{2}}{I_{1}-I_{3}}\right]
$$

By storing the arctan values in a look-up table and using the real-time subtracting capabilities of our image processing board we obtain the wavefront in a few seconds. If measurements with higher accuracy are required, an algorithm with five interferograms is more appropriate, because it is less sensitive to phase shift errors [19]. 
In this case five intensity distributions are measured. For a phase shift of $\Delta=\frac{\pi}{2}$ the phase can be calculated by :

$$
\varphi=\arctan \left[\frac{2\left(\mathrm{I}_{2}-\mathrm{I}_{4}\right)}{2 \mathrm{I}_{3}-\mathrm{I}_{5}-\mathrm{I}_{1}}\right]
$$

Figure 4 shows an example of a fringe analysis. Figure 4(A) shows the interference pattern of a steep aspheric surface measured with a CGH. The calculated phase of the wavefront is shown in a grey-scale map (B), as a pseudo 3D-plot (C) and as contour lines (D).

The deviation of the evaluated wavefront from a plane contains both surface and adjustment errors. A compensation of adjustment errors can be done by combining fringe analysis and ray tracing [15]. The measured wavefront is approximated by Zernike polynomials to have an interface to the ray tracing program. The actual adjustment state is investigated by varying the system parameters in such a way that the wavefront calculated by ray tracing has minimum deviations from the measured one.

\section{$\underline{\text { 5.Conclusions }}$}

An interferometric method for testing aspherical surfaces with the aid of computer generated holograms has been described. The combination of CGH's with partially compensating lenses is a very flexible technique, which allows the test of a wide range of asphericities. An important advantage of the synthetic holograms is the ability of absolute testing of surfaces. The digital phase measurement interferometry leads to a short measuring time and high accuracy.

\section{References}

1. Wyant, J.C.;Bennett, V.P. : Using computer generated holograms to test aspheric wavefronts. Appl. Opt. 11 (1972) 2833.

2. Fercher, A.F.; Kriese, M. : Binäre synthetische Hologramme zur Prüfung asphärischer optischer Elemente. Optik $\underline{35}$ (1972) 168.

3. Foulde, M.; Fercher, A.F.; Torge, R.; Wilson, R.N. : Optical testing by means of synthetic holograms and partial lens compensation. Opt. Commun. $\underline{7}$ (1973) 363

4. Wyant, J.C.; O'Neill, P.K. : Computer generated hologram ; Null lens test of aspheric wavefronts. Appl. Opt. 13 (1974) 2762.

5. Sirohi, R.S.; Blume, H.; Rosenbruch, K.-J. : Optical testing using synthetic holograms. Opt. Acta $\underline{23}$ (1976) 229. 
6. Fercher, A.F. : Computer generated holograms for testing optical elements : Error analysis and error compensation. Opt. Acta $\underline{23}$ (1976) 347

7. Handojo, A.; de Jong, J. : Interferometer for optical testing with computer-generated holograms. Appl. Opt. 16 (1977) 546

8. Yatagai, T.; Saito, H. : Interferometric testing with computer generated holograms : Aberration balancing method and error analysis. Appl. Opt. 17 (1978) 558

9. Schwider, J.; Burow, R.; Grzanna, J. : CGH-Testing of rotational symmetric aspheric in compensated interferometers. Opt. Appl. 2 (1979) 39

10. Schwider, J.; Burow , R.: Wave aberration caused by by misalignments of aspherics and their elimination. Opt. Appl. 9 (1979) 33

11. Tiziani, H. : Prospects of testing aspheric surfaces with computer-generated holograms. Proc SPIE 235 (1980) 72

12. Schwider, J.; Grzanna, J.; Spolaczyk, R.; Burow, R. :Testing aspherics in reflected light using blazed synthetic holograms. Opt. Acta $\underline{27}$ (1980) 683

13. Larionov, N.P.; Lukin, A.V.; Rafikov, R.A. :Testing of aspheric surfaces by means of axial synthesized holograms. Sov. J. Opt. Technol. 47 (1980) 667

14. Mercier, R.; Lowenthal, S. : Comparison of in-line and carrier frquency holograms in aspherics testing. Opt. Commun. $\underline{33}$ (1980) 251

15. Dörband, B.; Tiziani, H. : Testing aspheric surfaces with computer generated holograms : analysis of adjustment and shape errors. Appl. Opt. 24 (1985) 2604

16. Koliopoulis, C.L. : Interferometric optical phase measurement techniques. Ph. D. Dissertation University of Arizona, Tucson (1981)

17. Schwider, J.; Burow, R.; Elssner, K.-E.; Grzanna, J.; Spolaczyk, R.; Merkel, K. : Digital wave-front measuring interferometry : some systematic error sources. Appl. Opt. $\underline{22}$ (1983) 3421

18. Creath, K. : Comparison of phase shift algorithms. Proc. SPIE 680 (1986)

19. Hariharan, P.; Oreb, B.F.; Eijn, T. : Digital phase shifting interferometry : a simple error-compensating phase calculation algorithm. Appl. Opt. 26 (1987) 2504 


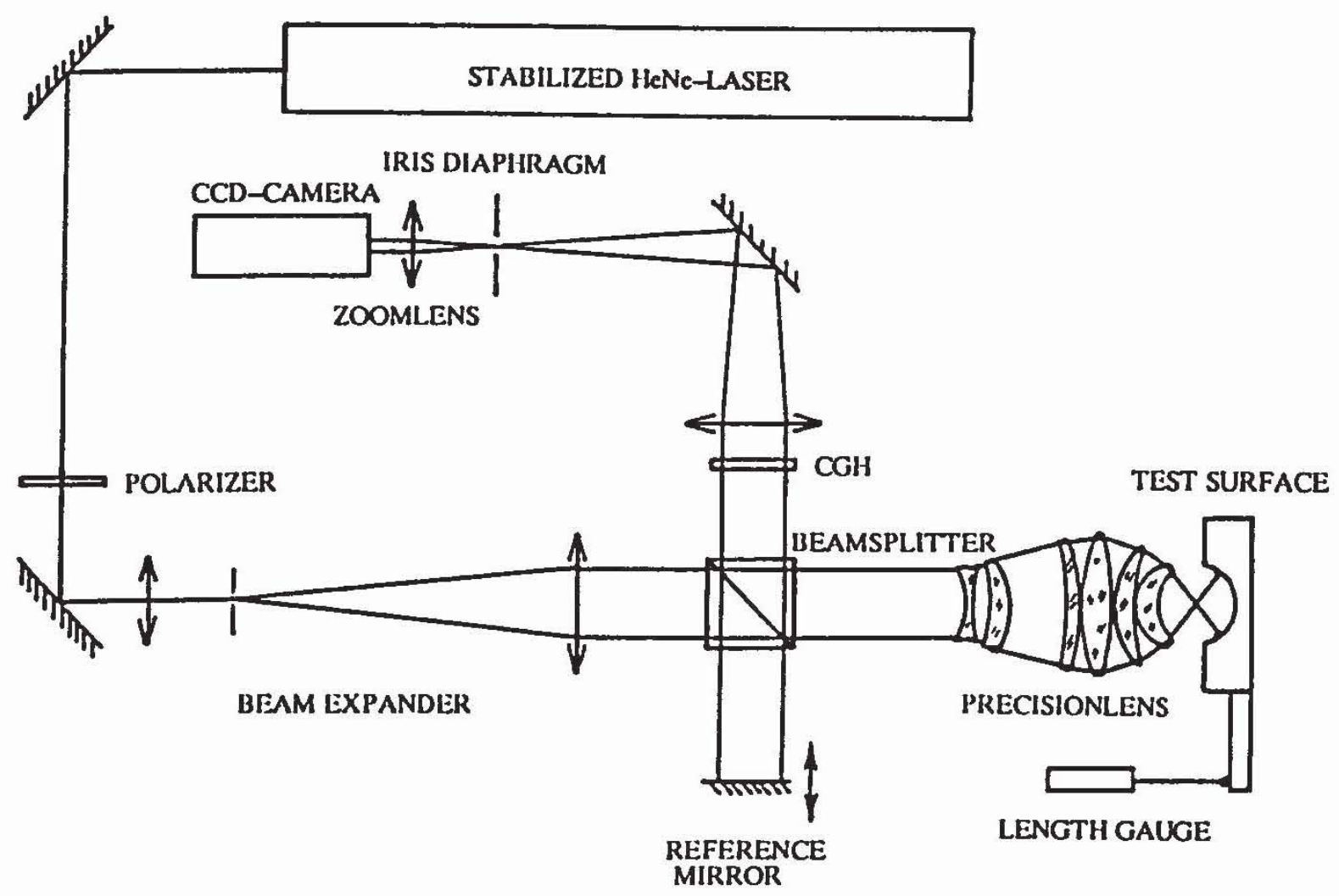

Fig.1. Twyman-Green interferometer with $\mathrm{CGH}$

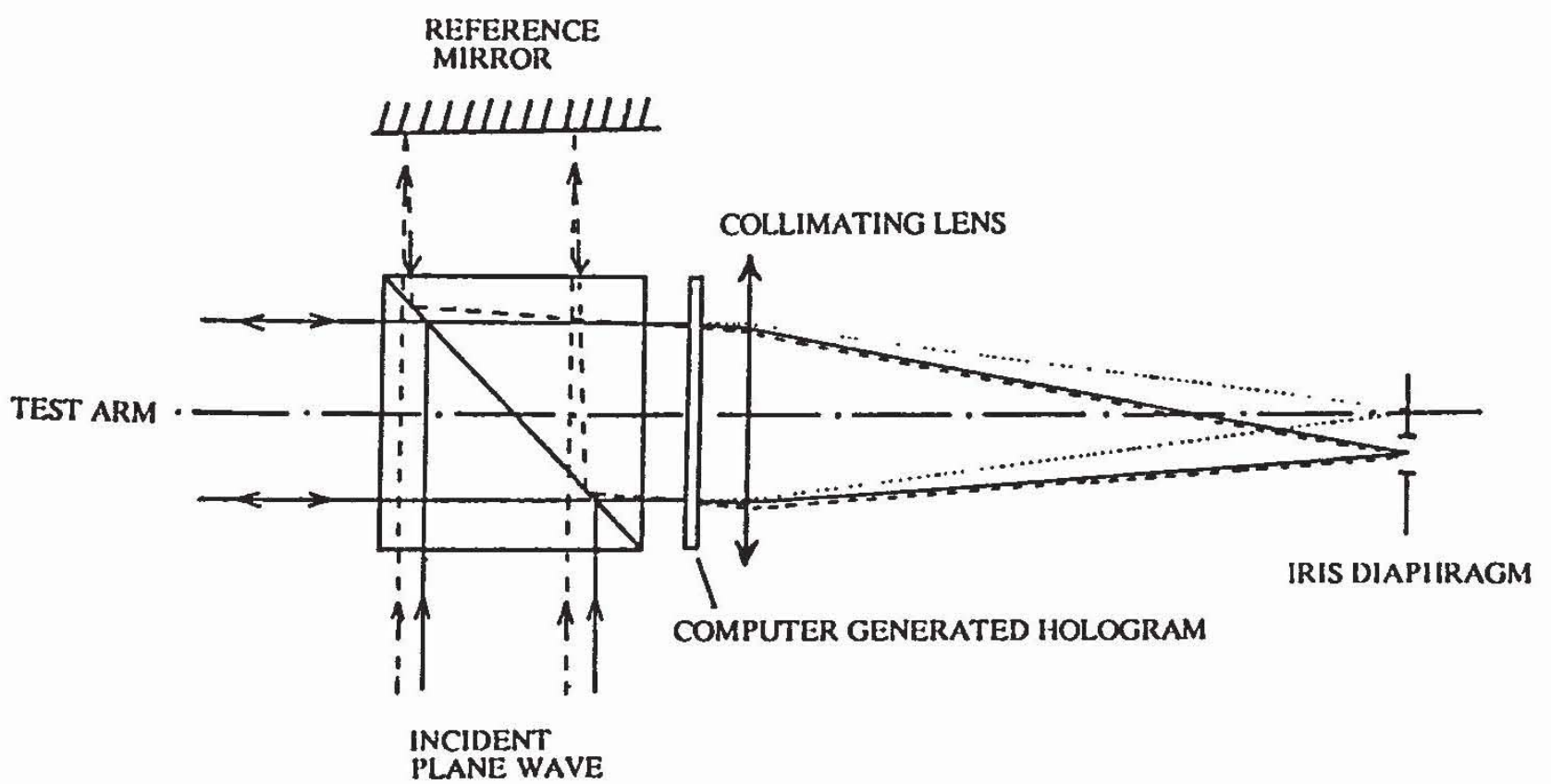

Fig.2. Superposition of diffracted waves. First diffraction order of test wave is superimposed with zero order reference wave. The other diffraction orders are blocked by an iris diaphragm. 


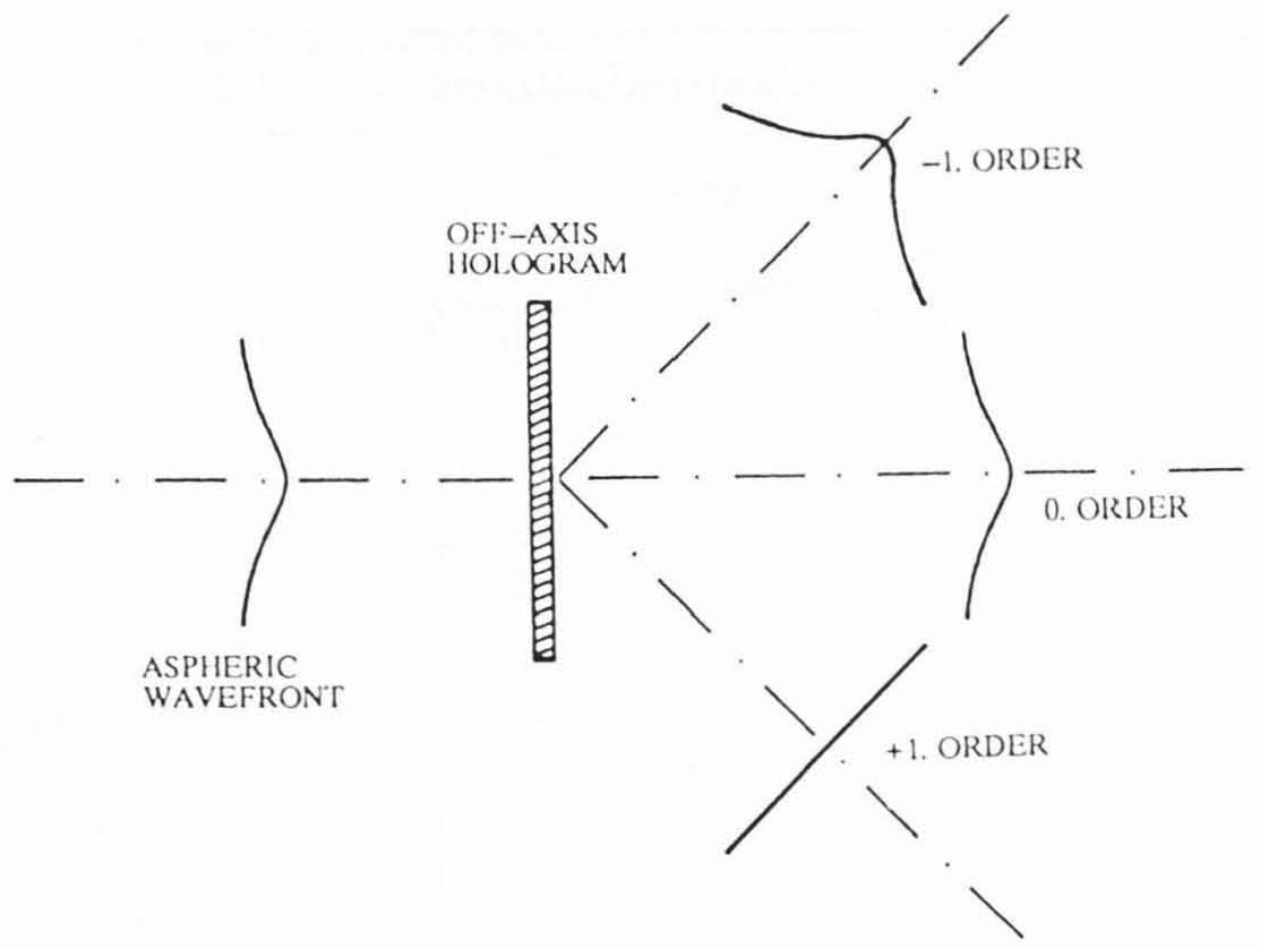

Fig.3. Diffraction of an aspheric wavefront by a CGH

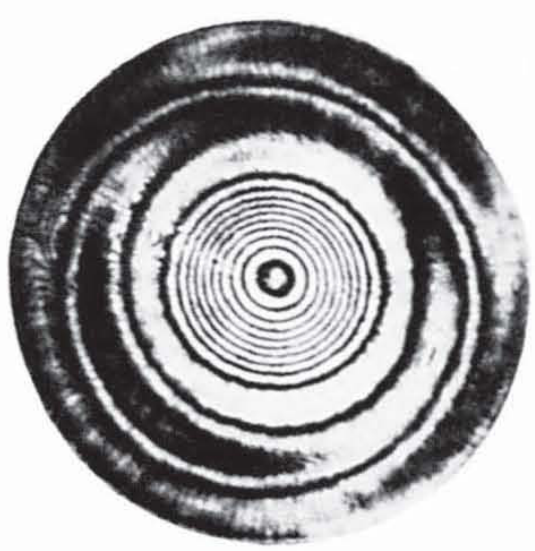

A

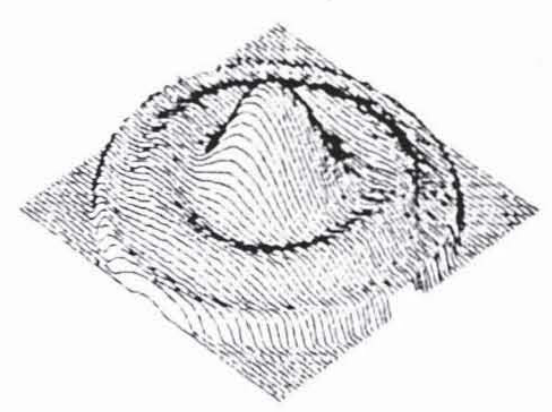

C

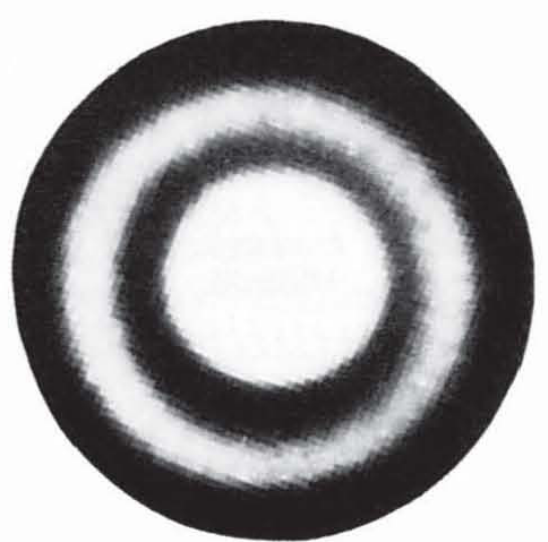

B

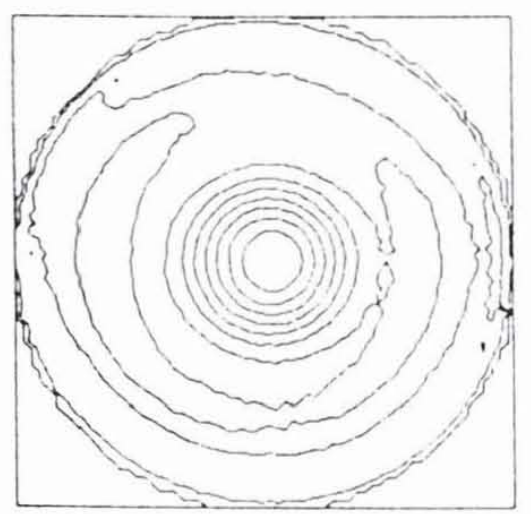

D

Fig.4. Fringe analysis of an asheric wavefront. Interferogram (A) and calculated wavefront coded in three different presentations:

grey level (B), pseudo 3-D (C), contour lines 